\title{
Stimulus intensity effects between and within subjects in auditory reaction time: A variable criterion analysis
}

\author{
G. ROBERT GRICE, ROBERT NULLMEYER, and V. ALAN SPIKER \\ University of New Mexico, Albuquerque, New Mexico 87131
}

\begin{abstract}
In simple auditory reaction time, Grice and Hunter (1964) obtained a significant interaction between signal intensity and the manner of intensity manipulation-between or within subjects. In a reanalysis by more recently developed methods of variable criterion theory, estimates of criterion level have been obtained for each subject in each condition. Criterion level was significantly higher in the within-subjects condition. Also, subjects receiving only the weak stimulus had lower criterion levels than those receiving only the strong stimulus. Both of these conditions provide an explanation of the interaction in terms of variable criterion theory.
\end{abstract}

Grice and Hunter (1964) found that the magnitude of the effect of stimulus intensity depended upon the manner in which the intensity variable was manipulated. If the manipulation was within subjects, each subject receiving two intensities in an irregular order, the intensity effect was greater than if the manipulation was between subjects, each subject receiving only one of the intensities. This result was obtained both for conditioned stimulus intensity in human eyelid conditioning and for signal intensity in simple auditory reaction time. Although originally interpreted in terms of adaptation level concepts, the finding subsequently led Grice (1968) to the development of variable criterion theory. According to this view, the excitatory strength leading to response evocation grows as a deterministic function of time following stimulus onset until it reaches a criterion value. At that time response evocation occurs. The criterion value is a random variable, normally distributed over trials. The mean level and variability of the criterion distribution are determined by variables influencing attention, adaptation, set, and motivation. Variable criterion theory not only provided a theoretical account of the Grice and Hunter (1964) result, but also led to a number of other theoretical developments and methods of data analysis in the areas of human conditioning and reaction time.

The theory provides two ways of accounting for the obtained interaction between the intensity effect and the method of stimulus presentation. First, criterion level may be affected by the intensity of the stimuli presented, such that subjects receiving only a weak stimulus may adopt a lower criterion than those receiving only a strong stimulus. On the other hand, subjects

This research was supported by Public Health Service Grant MH16400 from the National Institute of Mental Health. Requests for reprints should be sent to G. Robert Grice, Department of Psychology, University of New Mexico, Albuquerque, New Mexico 87131. receiving both in an irregular order must respond to both with the same mean criterion level. Grice (1968) pointed out that this was sufficient to account for the Grice and Hunter (1964) conditioning data. The second principle requires that the functions for the growth of excitatory or sensory strength for the different intensities diverge in the time dimension. Under this condition, the difference in reaction time (RT) dependent upon intensity will increase with increasing criterion levels. The RT data indicated that this effect might be involved since RTs in the within-subjects condition were significantly slower to both stimuli, indicating a larger difference in RT. Since Grice's (1968) initial presentation, methods have been further developed; a more complete analysis is now made possible by obtaining estimates of the criterion parameters for each subject. Such a reanalysis of the Grice and Hunter data is now especially desirable, since Grice, Nullmeyer, and Schnizlein (1979) failed to find a similar interaction in simple RT for visual intensity. The Grice and Hunter data are not ideal for such an analysis, since the number of trials was rather small (50 to each stimulus in the within condition and 100 in the between condition). The numbers used by Grice et al. (1979) were 120 and 240 . However, reasonable parameter estimates can be obtained, and the results of the analysis are presented here. ${ }^{1}$

Details of methods for estimating the mean $(\overline{\mathrm{C}})$ and standard deviations $(\sigma)$ of the criterion distributions for individual subjects have been presented elsewhere (Grice, Nullmeyer, \& Spiker, 1977; Grice et al., 1979). The first step was to obtain estimates of the functions for the growth of sensory strength or detection information $(V)$ from the more reliable (100-trial) data of the soft-only $(40 \mathrm{~dB})$ and loud-only $(100 \mathrm{~dB})$ groups. The method was the between-subjects scaling procedure used by Grice et al. (1979) and the results were mean within-subjects functions when the data of all subjects in each group were on common scales. The functions 
Table 1

Means (M) and Standard Deviations (SD) of the Theoretical Variables, Criterion Mean $(\bar{C})$, and Standard Deviation $(\sigma)$ in Each Condition

\begin{tabular}{|c|c|c|c|c|c|c|c|c|}
\hline \multirow[b]{3}{*}{ Condition } & \multicolumn{4}{|c|}{$\overline{\mathrm{C}}$} & \multicolumn{4}{|c|}{$\sigma$} \\
\hline & \multicolumn{2}{|c|}{ Loud } & \multicolumn{2}{|c|}{ Soft } & \multicolumn{2}{|c|}{ Loud } & \multicolumn{2}{|c|}{ Soft } \\
\hline & $\mathbf{M}$ & SD & M & SD & $\mathbf{M}$ & SD & $\mathbf{M}$ & SD \\
\hline $\begin{array}{l}\text { Within } \\
\text { Between }\end{array}$ & $\begin{array}{r}.000 \\
-.696\end{array}$ & $\begin{array}{l}.672 \\
.975\end{array}$ & $\begin{array}{r}.000 \\
-1.306\end{array}$ & $\begin{array}{r}.622 \\
1.071\end{array}$ & $\begin{array}{l}.968 \\
.815\end{array}$ & $\begin{array}{l}.257 \\
.213\end{array}$ & $\begin{array}{r}.958 \\
1.047\end{array}$ & $\begin{array}{l}.300 \\
.301\end{array}$ \\
\hline
\end{tabular}

turned out to be typically negatively accelerated and were fitted with exponential growth functions. The fits were adequate, with the proportions of variance accounted for by the calculated equations being .994 for loud and .999 for soft. The function for loud grew more rapidly than that for soft, and, within the range of responding, they continuously diverged in the time dimension in the manner described by Grice et al. (1979) as characteristic of auditory data.

All criterion parameter estimates were made with respect to the calculated functions. For the loud and soft conditions in the within-subjects condition, estimates of $\overline{\mathrm{C}}$ and $\sigma$ were obtained from the intercepts and slopes of linear response evocation characteristics (RECs) relating the inverse normal transformation of each cumulative distribution to the calculated functions at corresponding time intervals. Assuming the loud and soft estimates to be from a common criterion distribution, all estimates were transformed to a scale with the origin at the mean of the subject means and the unit $(\sigma)$ based on the mean of the within-subjects variances. The two functions for the growth of $\mathrm{V}$ were then transformed to the scale, and the values of $\overline{\mathrm{C}}$ and $\sigma$ for the loud-only and soft-only groups were determined with respect to the transformed functions. ${ }^{2}$ Thus, all parameters were on a common scale based on the withinsubjects criterion distribution. The validity of the estimates may be evaluated in terms of the linearity of the 80 least-squares regression solutions relating the subject data to the $\mathrm{V}$ functions. For the between condition, the mean value of $\mathrm{r}^{2}$ was .988 and the median was .991 . For the within condition, the mean was .981 and the median was .987.

The means and standard deviations of the value of $\overline{\mathrm{C}}$ and $\sigma$ are presented in Table 1 . As indicated by $\overline{\mathrm{C}}$, the levels of the criterion were significantly lower for both intensities in the between-subjects condition [for loud,

Table 2

Intercorrelation of the Means and Standard Deviations (SD) of Subject RTs and the Theoretical Parameters, Criterion Mean $(\overline{\mathbf{C}})$, and Criterion Standard Deviation $(\sigma)$

\begin{tabular}{llll}
\hline & SD & $\overline{\mathrm{C}}$ & $\sigma$ \\
\hline Mean & .530 & .942 & -.443 \\
SD & & .416 & .357 \\
$\overline{\mathrm{C}}$ & & & -.450 \\
\hline
\end{tabular}

$\mathrm{t}(38)=2.60, \mathrm{p}<.02$; for soft, $\mathrm{t}(38)=4.71, \mathrm{p}<.001]$. The data also support the prediction that the soft-only group would have lower criteria than the loud-only group $[\mathrm{t}(38)=1.88, \mathrm{p}<.05$, one tail]. Thus, both of the factors identified by variable criterion theory as leading to an interaction between the intensity effect and method of presentation were present in the data. These are higher criterion levels in the within condition in the presence of temporally diverging sensory functions, and the dependence of criterion level upon intensity in the between condition. Neither of these factors was present in the visual data of Grice et al. (1979). Not only were there no significant effects of the experimental variables upon criterion level, but the visual sensory functions were nearly parallel within the range of most responding. This comparison should contribute to the understanding of the fact that, while interactions of intensity and criterion effects are common in auditory RT studies, they are rare with the visual modality (Nissen, 1977).

Differences in criterion varability, $\sigma$, are small in Table 2, but the difference between loud only and soft only reached significance $[\mathrm{t}(38)=2.38, \mathrm{p}<.05]$. This is possibly due to a negative correlation between $\mathrm{C}$ and $\sigma$.

As a final analysis, the intercorrelations of subject RT means and standard deviations (SD) with the theoretical criterion parameters are presented in Table 2. These are within-conditions correlations based upon Ns of 80 . The positive correlation between the mean and $\mathrm{SD}$ is an implication of the negatively accelerated growth of sensory strength, $\mathrm{V}$. The high correlation between the mean and $\overline{\mathrm{C}}$ is also to be expected. It is seen that RT variability, SD, is determined by both $\overline{\mathrm{C}}$ and $\sigma$. The positive relation with $\overline{\mathrm{C}}$ is also an implication of the negatively accelerated growth of $\mathrm{V}$. The negative correlation between $\overline{\mathrm{C}}$ and $\sigma$ was also observed by Grice et al. (1979) for the visual data. It implies a form of tradeoff in which low criteria are achieved at some cost in criterion variability. The negative relation between $\sigma$ and mean RT reflects this effect.

\section{REFERENCES}

Erlebacher, A. Design and analysis of experiments contrasting the within and between subjects manipulation of the independent variable. Psychological Bulletin, 1977, 84, 212-219.

Grice, G. R. Stimulus intensity and response evocation. Psychological Review, 1968, 75, 359-373. 
Grice, G. R., \& Hunter, J. J. Stimulus intensity effects depend upon the type of experimental design. Psychological Review, 1964, 71, 247-256.

Grice, G. R., Nullmeyer, R., \& Schnizlein, J. M. Variable criterion analysis of brightness effects in simple reaction time. Journal of Experimental Psychology: Human Perception and Performance, 1979, 5, 303-314.

Grice, G. R., Nullmeyer, R., \& Spiker, V. A. Application of variable criterion theory to choice reaction time. Perception \& Psychophysics, 1977, 22, 431-449.

Nissen, M. J. Stimulus intensity and information processing. Perception \& Psychophysics, 1977, 22, 338-352.

\section{NOTES}

1. Preliminary to the present theoretical analysis, we reanalyzed the Grice and Hunter (1964) RT data with a more sensitive analysis of variance design now available (Erlebacher, 1977). The design utilizes all of the data of the within-subjects condition rather than half, as originally. Thus the $\mathrm{N}$ was 20 in each cell. The reanalysis merely confirmed the pattern of significance originally reported.

2. Expressed on the scale of the within-subjects condition, the two sensory functions are as follows:

$$
V_{L}(t)=2.098-85.544 e^{-.01158 t}
$$

and

$$
\mathrm{V}_{\mathrm{S}}(\mathrm{t})=2.295-58.570 \mathrm{e}^{-.00801 \mathrm{t}} \text {, }
$$

where $V_{L}(t)$ and $V_{S}(t)$ are the values of the loud and soft functions at time $t$, following stimulus onset, with $t$ in milliseconds.

In the course of the present reanalysis, an error was discovered in Grice and Hunter's (1964) graphic presentation of the RTs. Labels on the time ordinate are $60 \mathrm{msec}$ too long. Correction for a constant $60-\mathrm{msec}$ lag in the recording equipment was not made in constructing the figure. Equations 1 and 2 are for the correct values.

(Received for publication June 8, 1979.) 